\title{
C. Merkmale der Beziehungen zwischen der Schweiz und den Entwicklungsländern
}

Gérard Perroulaz and Xavier Tschumi Canosa

\section{(2) OpenEdition}

1 Journals

Electronic version

URL: http://journals.openedition.org/sjep/202

DOI: $10.4000 /$ sjep.202

ISSN: 1663-9677

Publisher

Institut de hautes études internationales et du développement

Printed version

Date of publication: 1 avril 2005

Number of pages: $236-250$

ISBN: 2-88247-058-4

ISSN: $1660-5926$

\section{Electronic reference}

Gérard Perroulaz und Xavier Tschumi Canosa, «C. Merkmale der Beziehungen zwischen der Schweiz und den Entwicklungsländern », Schweizerisches Jahrbuch für Entwicklungspolitik [Online], 24-1 | 2005, Online erschienen am: 22 April 2010, abgerufen am 08 September 2020. URL : http:// journals.openedition.org/sjep/202 ; DOI : https://doi.org/10.4000/sjep.202 


\section{Merkmale der Beziehungen zwischen der Schweiz und den Entwicklungsländern}

\section{C.1. Hauptcharakteristiken}

Die einführenden Tabellen 1 bis 3 geben einen Überblick über die Beziehungen der Schweiz zu sämtlichen Entwicklungs- und Transitionsländern (siehe Länderliste gemäss DAC-Klassifikation am Schluss des Jahrbuchs). Anhand der oben erwähnten Tabellen lassen sich folgende Merkmale dieser Beziehungen herausstellen:

$\square$ Die Beiträge des Privatsektors sind im Grossen und Ganzen viel höher als die Leistungen der öffentlichen und privaten Hilfe. So beläuft sich die bilaterale öffentliche Hilfe für die Entwicklungsländer im Jahr 2003 auf 1271 Millionen Franken und die Hilfe der Nichtregierungsorganisationen (NRO) auf 377 Millionen Franken, wohingegen die Direktinvestitionen im Ausland 2,8 Milliarden Franken erreichen. Die Wirtschaftsbeziehungen mit den Transitionsländern im gleichen Jahr sind noch bedeutender: Die Transitionsländer erhielten 90,9 Millionen Franken an öffentlicher Entwicklungshilfe und 15,4 Millionen Franken an Hilfe der NRO, wogegen sich die Investitionen auf 1,6 Milliarden Franken beliefen.

$\square$ Die geografische Aufteilung zwischen öffentlicher Hilfe und privaten Investitionsströmen ist sehr verschieden. Die öffentliche Entwicklungshilfe und die Hilfe der NRO sind hauptsächlich für die einkommensschwachen Länder bestimmt, während die Investitionen vor allem auf die Länder mit mittlerem Einkommen und die mitteleuropäischen Staaten ausgerichtet sind, und die Ausfuhren in die Länder mit mittlerem Einkommen, die mittel- und osteuropäischen Staaten und die fortgeschrittenen Entwicklungsländer gehen.

๖ Die Situation der afrikanischen Staaten ist interessant zu erwähnen. Die afrikanischen Länder erhalten 27 Prozent der bilateralen öffentlichen Entwicklungshilfe und ein Drittel der Hilfe der NRO, jedoch machen sie nur 20 Prozent der Einfuhren der Schweiz aus den Entwicklungsländern und 12 Prozent der Ausfuhren aus. Die Nettoinvestitionen sind sogar negativ, was eine Kapitalrückführung (Desinvestition) darstellt.

๑ Die Situation der 49 am wenigsten fortgeschrittenen Länder (LDC) ist ähnlich. Während die am wenigsten entwickelten Länder im Jahr 200327 Prozent der gesamten öffentlichen Entwicklungshilfe und 40 Prozent der Hilfe der NRO erhielten, kamen lediglich 1,9 Prozent der Einfuhren aus den Entwicklungsländern aus den LDC, und die Netto-Investitionsströme machten nur 0,8 Prozent der gesamten Investitionen in die Entwicklungsländer aus. Auf die am wenigsten entwickelten Länder fallen lediglich 0,12 Prozent der Gesamteinfuhren der Schweiz.

$\square$ Die Netto-Finanzflüsse zwischen den Entwicklungsländern und der Schweiz fallen im Allgemeinen zugunsten der Schweiz aus. Berücksichtigt man lediglich die Flüsse der öffentlichen und privaten Hilfe, die Investitionen und den 
Handelssaldo mit bestimmten Entwicklungsgebieten, so stellt man fest, dass die Flüsse aus diesen Regionen in die Schweiz die Hilfeleistungen der Schweiz in diese Gebiete übersteigen. Diese Angaben sind partiell, da gewisse Flüsse, die schwer nach Länderkategorien aufzuteilen sind - wie die Bankflüsse (Schuldendienst und -rückzahlung, Nettoanlagen in Schweizer Banken) oder die Überweisungen der Migrantinnen und Migranten in der Schweiz an ihre Familien - nicht berücksichtigt werden.

$\checkmark$ Die bilaterale öffentliche Hilfe des Bundes und die (aus privaten Mitteln finanzierte) Hilfe der schweizerischen NRO haben eine ziemlich ähnliche geografische Aufteilung. Jedoch entfällt bei der Hilfe der NRO ein grösserer Anteil auf die Entwicklungsländer Asiens, Afrikas und Lateinamerikas.

\section{C.1.1. Platz der Entwicklungsländer und Transitionsländer im Aussenhandel der Schweiz (Tabellen von Teil B).}

Die Gesamteinfuhren der Schweiz betrugen im Jahr 2003 129,7 Milliarden Franken. Gemäss der Länderklassifikation des DAC erreichten die Einfuhren der Schweiz aus den Entwicklungsländern 8,2 Milliarden Franken (d.h. 6,3\% der Gesamtimporte) und die Einfuhren aus den Transitionsländern 7,5 Milliarden Franken. Lediglich 12,1 Prozent der Einfuhren kommen aus den Entwicklungs- und Transitionsländern, während 23 Prozent der Ausfuhren in diese Länder gehen.

\section{Grafik 1: Entwicklungs- und Transitionsländer, mit denen der Handelsüberschuss am höchsten ist, 2003}

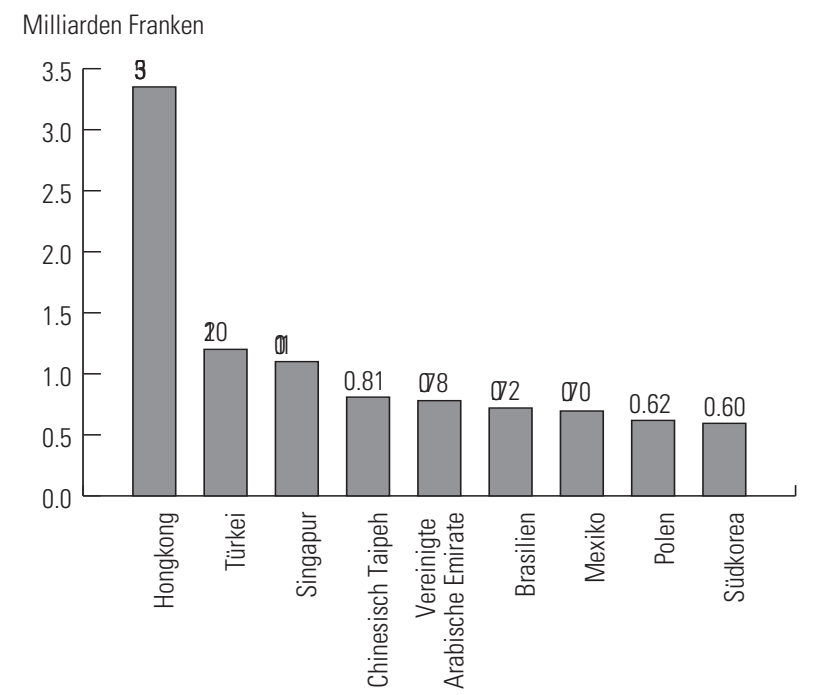

Quelle: Eidg. Oberzolldirektion, Schweizerische Aussenhandelsstatistik, Statistik nach Waren und Ländern, Bern.

Die Schweiz verzeichnet mit den Entwicklungs- und Transitionsländern zumeist eine überschüssige Handelsbilanz, wogegen die Handelsbilanz mit den Industrieländern im Allgemeinen defizitär ist (vgl. die drei letzten Spalten der Tabellen von Teil B). So importierte die Schweiz im Jahr 2003 beispielsweise für 
41,9 Milliarden Franken Produkte aus Deutschland und exportierte für 28,2 Milliarden Franken in dieses Land, was ein Handelsdefizit von 13,7 Milliarden Franken ergibt. Der Handelsüberschuss mit sämtlichen Entwicklungsländern betrug 2003 7,1 Milliarden Franken. Der Handelsüberschuss mit den fortgeschrittenen Entwicklungsländern erreichte 6,9 Milliarden Franken und mit den mittel- und osteuropäischen Staaten 1,4 Milliarden Franken.

Der Fall Chinas ist interessant zu erwähnen, da die Schweiz in den letzten Jahren viel mehr aus China importierte, als sie in dieses Land exportierte, mit einem zurückgehenden negativen Handelssaldo. 2003 ist der Handelssaldo leicht positiv geworden.

\section{C.2. Aussenhandel der Schweiz}

\section{C.2.1. Übersicht}

\section{Schema 1: Aussenhandel mit den Entwicklungs- und Transitionsländern, 2003} (in Millionen Franken)

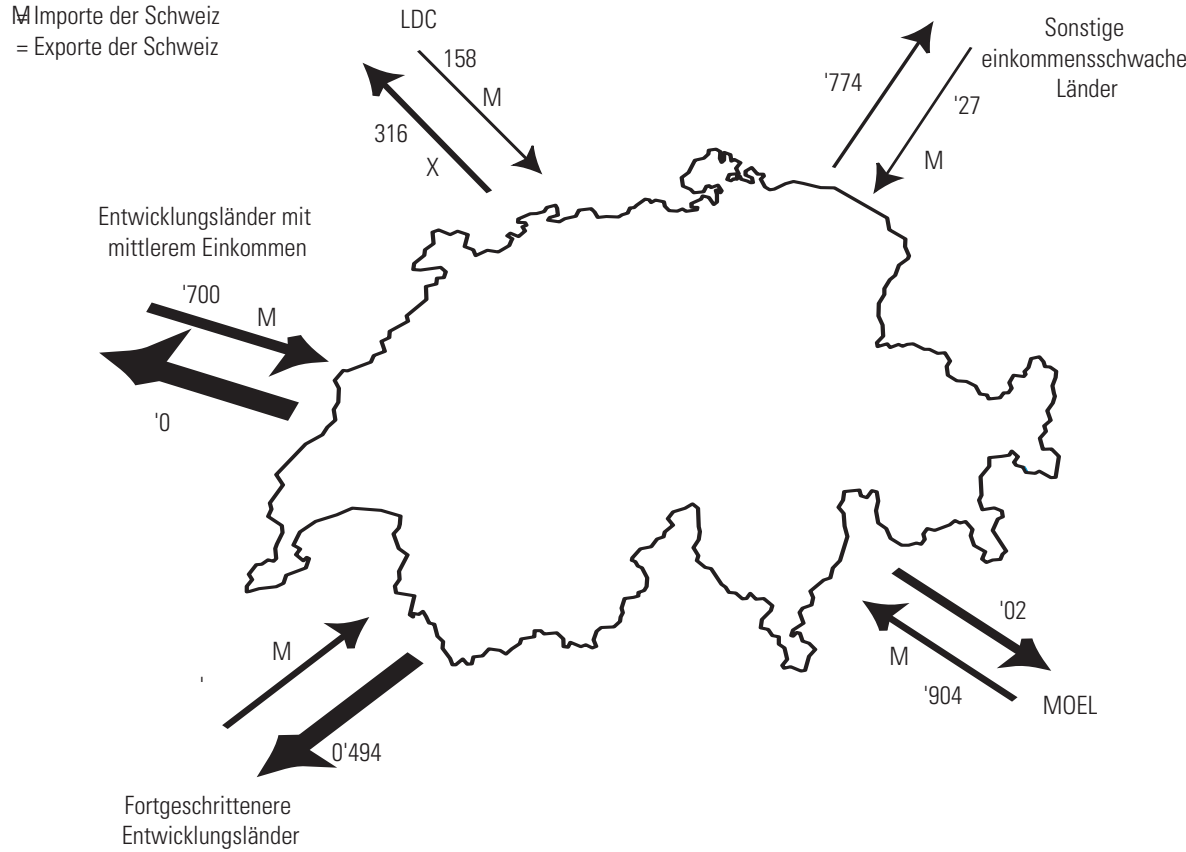

Quelle: Angepasst aus der Publikation: Aus Fehlern lernen? Die Schweiz und die Dritte Welt, Richard Gerster, Verlag Ex Libris, Zürich, S. 65; Eidg. Oberzolldirektion, Schweizerische Aussenhandelsstatistik, 2004, S. 869-871 betreffend die aktualisierten Zahlen.

Die Schweiz unterhält vor allem mit den Nachbarländern und den Industriestaaten Handelsbeziehungen: 58 Prozent ihrer Einfuhren kommen aus den angrenzenden Ländern und 44,5 Prozent ihrer Ausfuhren gehen in diese Länder. Wie aus Tabelle 4 ersichtlich, sind unter den zwanzig wichtigsten Bezugsländern der Schweiz nur 
fünf Entwicklungs- oder Transitionsländer und unter den zwanzig wichtigsten Absatzländern der Schweiz nur acht Entwicklungs- oder Transitionsländer.

Tabelle 4: Die zwanzig wichtigsten Handelspartner der Schweiz, 2003

\begin{tabular}{|c|c|c|c|c|c|c|c|}
\hline \multicolumn{4}{|c|}{ Wichtigste Bezugsländer } & \multicolumn{4}{|c|}{ Wichtigste Absatzländer } \\
\hline \multicolumn{4}{|c|}{ Einfuhren } & \multicolumn{4}{|c|}{ Ausfuhren } \\
\hline Land $^{\mathrm{a}}$ & Mio Fr. & $\%$ & $\sum \%$ & Land ${ }^{\mathrm{a}}$ & Mio Fr. & $\%$ & $\sum \%$ \\
\hline 1. Deutschland & $41^{\prime} 895.5$ & 32.3 & 32.3 & 1. Deutschland & $28^{\prime} 217.3$ & 20.8 & 20.8 \\
\hline 2. Frankreich & $13^{\prime} 983.3$ & 10.8 & 43.1 & 2. USA & $14^{\prime} 943.4$ & 11.0 & 31.9 \\
\hline 3. Italien & $13^{\prime} 854.0$ & 10.7 & 53.7 & 3. Frankreich & $11^{\prime} 815.1$ & 8.7 & 40.6 \\
\hline 4. USA & 6'634.8 & 5.1 & 58.9 & 4. Italien & $11^{\prime} 186.0$ & 8.3 & 48.9 \\
\hline 5. Niederlande & $6^{\prime} 475.1$ & 5.0 & 63.9 & 5. Grossbritannien & $6^{\prime} 582.1$ & 4.9 & 53.7 \\
\hline 6. Österreich & $5^{\prime} 487.4$ & 4.2 & 68.1 & 6. Japan & $5^{\prime} 405.7$ & 4.0 & 57.7 \\
\hline 7. Grossbritannien & $5^{\prime} 260.2$ & 4.1 & 72.1 & 7. Spanien & $4^{\prime} 744.0$ & 3.5 & 61.2 \\
\hline 8. Irland & $4^{\prime} 794.1$ & 3.7 & 75.8 & 8. Österreich & $4^{\prime} 469.4$ & 3.3 & 64.5 \\
\hline 9. Belgien & $33^{\prime} 943.0$ & 3.0 & 78.9 & 9. Niederlande & $4^{\prime} 402.1$ & 3.3 & 67.8 \\
\hline 10. Spanien & $33^{\prime} 040.5$ & 2.3 & 81.2 & 10. Hongkong & $4^{\prime} 002.2$ & 3.0 & 70.7 \\
\hline 11. Japan & $2 ' 728.5$ & 2.1 & 83.3 & 11. Belgien & $2{ }^{\prime} 649.4$ & 2.0 & 72.7 \\
\hline 12. China & $2^{\prime} 413.9$ & 1.9 & 85.2 & 12. China & $2^{\prime} 460.0$ & 1.8 & 74.5 \\
\hline 13. Schweden & 1'660.8 & 1.3 & 86.5 & 13. Türkei & $1^{\prime} 650.2$ & 1.2 & 75.7 \\
\hline 14. Russland & 1'175.6 & 0.9 & 87.4 & 14. Schweden & 1'620.2 & 1.2 & 76.9 \\
\hline 15. Dänemark & 1'057.8 & 0.8 & 88.2 & 15. Kanada & 1'428.9 & 1.1 & 78.0 \\
\hline 16. Tschechische Re & p. 882.8 & 0.7 & 88.9 & 16. Singapur & 1'358.7 & 1.0 & 79.0 \\
\hline 17. Südafrika & 862.2 & 0.7 & 89.5 & 17. Taiwan & 1'329.7 & 1.0 & 80.0 \\
\hline 18. Finnland & 797.3 & 0.6 & 90.1 & 18. Brasilien & 1'163.6 & 0.9 & 80.8 \\
\hline 19. Libyen & 755.9 & 0.6 & 90.7 & 19. Saudi-Arabien & 1'123.7 & 0.8 & 81.6 \\
\hline 20. Ungarn & 711.2 & 0.5 & 91.3 & 20. Polen & $1 ' 122.9$ & 0.8 & 82.5 \\
\hline Übrige Länder & $11 ' 328.9$ & 8.7 & 100.0 & Übrige Länder & $23^{\prime} 730.4$ & 17.5 & 100.0 \\
\hline Gesamtbetrag & $129^{\prime} 742.8$ & & 100.0 & Gesamtbetrag & 135 '405.0 & & 100.0 \\
\hline
\end{tabular}

Quelle: Eidg. Oberzolldirektion, Schweizerische Aussenhandelsstatistik, Statistik nach Waren und Ländern, Bern, 2004, S. 869-871.

a Im Fettdruck Länder auf der DAC-Liste der Entwicklungsländer (Teil I) oder auf der Liste der Transitionsländer (Teil II).

Wir führen ab diesem Jahr eine geografische Aufteilung des Aussenhandels anhand der Länderklassifikation des DAC ein. Somit sind die Tabellen 5 und 6 nicht mit den Tabellen des Teils „Handel“ der Vorjahre vergleichbar, bei denen wir die Länderklassifikation der UNCTAD angewandt hatten. Der Handel mit sämtlichen Entwicklungsländern und Transitionsländern ist in den drei letzten Spalten der Tabellen 1 bis 3 von Teil B der vorliegenden Statistik detailliert. 


\section{C.2.2. Entwicklungsländer und Transitionsländer}

Die Tabellen 5 und 6 führen die fünfzehn wichtigsten Handelspartner der Schweiz in der Gruppe der Entwicklungsländer und in der Gruppe der Transitionsländer auf.

Tabelle 5: Die fünfzehn wichtigsten Handelspartner der Schweiz unter den Entwicklungsländerna ${ }^{\mathrm{a}} 2003$

\begin{tabular}{|c|c|c|c|c|c|c|c|}
\hline \multicolumn{4}{|c|}{ Wichtigste Bezugsländer } & \multicolumn{4}{|c|}{ Wichtigste Absatzländer } \\
\hline \multicolumn{4}{|c|}{ Einfuhren } & \multicolumn{3}{|c|}{ Ausfuhren } & \\
\hline Land & Mio fr. & $\%^{\mathrm{b}}$ & $\%^{c}$ & Land & Mio fr. & $\%^{\mathrm{b}}$ & $\%$ c \\
\hline 1. China & $2 ' 413.9$ & 29.4 & 1.86 & 1. China & $2 ' 460.0$ & 16.1 & 1.82 \\
\hline 2. Südafrika & 862.2 & 10.5 & 0.66 & 2. Türkei & 1'650.2 & 10.8 & 1.22 \\
\hline 3. Thailand & 608.1 & 7.4 & 0.47 & 3. Brasilien & 1'163.6 & 7.6 & 0.86 \\
\hline 4. Indien & 500.2 & 6.1 & 0.39 & 4. Saudi-Arabien & 1'123.7 & 7.4 & 0.83 \\
\hline 5. Brasilien & 443.9 & 5.4 & 0.34 & 5. Mexiko & 914.0 & 6.0 & 0.68 \\
\hline 6. Türkei & 432.6 & 5.3 & 0.33 & 6. Thailand & 777.6 & 5.1 & 0.57 \\
\hline 7. Nigeria & 351.8 & 4.3 & 0.27 & 7. Indien & 741.6 & 4.9 & 0.55 \\
\hline 8. Mexiko & 218.6 & 2.7 & 0.17 & 8. Iran & 541.5 & 3.5 & 0.40 \\
\hline 9. Libanon & 190.8 & 2.3 & 0.15 & 9. Südafrika & 523.0 & 3.4 & 0.39 \\
\hline 10. Malaysia & 174.7 & 2.1 & 0.13 & 10. Malaysia & 449.4 & 2.9 & 0.33 \\
\hline 11. Saudi-Arabien & 173.8 & 2.1 & 0.13 & 11. Ägypten & 415.1 & 2.7 & 0.31 \\
\hline 12. Iran & 170.5 & 2.1 & 0.13 & 12. Indonesien & 289.4 & 1.9 & 0.21 \\
\hline 13. Indonesien & 167.1 & 2.0 & 0.13 & 13. Argentinien & 248.8 & 1.6 & 0.18 \\
\hline 14. Vietnam & 151.3 & 1.8 & 0.12 & 14. Pakistan & 245.5 & 1.6 & 0.18 \\
\hline 15. Algerien & 128.5 & 1.6 & 0.10 & 15. Kroatien & 213.7 & 1.4 & 0.16 \\
\hline $\begin{array}{l}\text { Übrige } \\
\text { Entwicklungsländer }\end{array}$ & 1'209.3 & 14.8 & 0.93 & $\begin{array}{l}\text { Übrige } \\
\text { Entwicklungsländer }\end{array}$ & 3'531.2 & 23.1 & 2.61 \\
\hline Gesamtbetrag & 8'197.3 & 100.0 & 6.32 & Total & $15^{\prime} 288.3$ & 100.0 & 11.29 \\
\hline
\end{tabular}

Quelle: Eidg. Oberzolldirektion, Schweizerische Aussenhande/sstatistik, 2004, S. 869-871.

a Entwicklungsländer gemäss Teil I der DAC-Liste (am Schluss des Jahrbuchs).

${ }^{\mathrm{b}}$ Anteil des Landes an den gesamten Einfuhren/Ausfuhren der Entwicklungsländer.

${ }^{c}$ Anteil des Landes an den gesamten Einfuhren/Ausfuhren aller Partnerländer der Schweiz. 
Tabelle 6: Die fünfzehn wichtigsten Handelspartner der Schweiz unter den Transitionsländern ${ }^{\mathrm{a}}$, 2003

\begin{tabular}{|c|c|c|c|c|c|c|c|}
\hline \multicolumn{4}{|c|}{ Wichtigste Bezugsländer } & \multicolumn{4}{|c|}{ Wichtigste Absatzländer } \\
\hline \multicolumn{4}{|c|}{ Einfuhren } & \multicolumn{4}{|c|}{ Ausfuhren } \\
\hline Land & Mio fr. & $\%^{\mathrm{b}}$ & $\%^{c}$ & Land & Mio fr. & $\%^{\mathrm{b}}$ & $\%^{c}$ \\
\hline 1. Russland & 1'175.6 & 15.6 & 0.91 & 1. Hongkong & 4'002.2 & 25.3 & 2.96 \\
\hline 2. Tschechische Rep. & 882.8 & 11.7 & 0.68 & 2. Singapur & 1'358.7 & 8.6 & 1.00 \\
\hline 3. Libyen & 755.9 & 10.0 & 0.58 & 3. Taiwan & 1'329.7 & 8.4 & 0.98 \\
\hline 4. Ungarn & 711.2 & 9.4 & 0.55 & 4. Polen & 1'122.9 & 7.1 & 0.83 \\
\hline 5. Hongkong & 648.7 & 8.6 & 0.50 & 5. Südkorea & 1'101.6 & 7.0 & 0.81 \\
\hline 6. Taiwan & 521.0 & 6.9 & 0.40 & 6. Russland & 1'064.7 & 6.7 & 0.79 \\
\hline 7. Südkorea & 507.7 & 6.7 & 0.39 & 7. Tschechische Rep. & 1'032.4 & 6.5 & 0.76 \\
\hline 8. Polen & 504.6 & 6.7 & 0.39 & 8. Verein. Arab. Emirate & te 908.5 & 5.8 & 0.67 \\
\hline 9. Israel & 370.9 & 4.9 & 0.29 & 9. Ungarn & 846.8 & 5.4 & 0.63 \\
\hline 10. Slowakei & 306.6 & 4.1 & 0.24 & 10. Israel & 626.6 & 4.0 & 0.46 \\
\hline 11. Singapur & 228.5 & 3.0 & 0.18 & 11. Rumänien & 305.2 & 1.9 & 0.23 \\
\hline 12. Slowenien & 173.0 & 2.3 & 0.13 & 12. Slowenien & 292.8 & 1.9 & 0.22 \\
\hline 13. Jungferninseln & 160.2 & 2.1 & 0.12 & 13. Slowakei & 283.4 & 1.8 & 0.21 \\
\hline 14. Rumänien & 142.2 & 1.9 & 0.11 & 14. Ukraine & 199.1 & 1.3 & 0.15 \\
\hline 15. Verein. Arab. Emirate & 128.3 & 1.7 & 0.10 & 15. Libyen & 122.3 & 0.8 & 0.09 \\
\hline $\begin{array}{l}\text { Übrige } \\
\text { Transitionsländer }\end{array}$ & 319.5 & 4.2 & 0.25 & $\begin{array}{l}\text { Übrige } \\
\text { Transitionsländer }\end{array}$ & 1'198.8 & 7.6 & 0.89 \\
\hline Gesamtbetrag & 7'536.7 & 100.0 & 5.81 & Gesamtbetrag $15^{\prime}$ & $5^{\prime} 795.7$ & 100.0 & 11.67 \\
\hline
\end{tabular}

Quelle: Eidg. Oberzolldirektion, Schweizerische Aussenhandelsstatistik, 2004, S. 869-871.

a Fortgeschrittenere Entwicklungsländer und mittel- und osteuropäische Länder gemäss Teil II der DAC-Liste (am Schluss des Jahrbuchs).

b Anteil des Landes an den gesamten Einfuhren/Ausfuhren der Transitionsländer.

c Anteil des Landes an den gesamten Einfuhren/Ausfuhren aller Partnerländer der Schweiz. 


\section{C.2.3. Fünf Beispiele zu von der Schweiz importierten Agrarprodukten}

Die folgende Tabelle führt die Angaben zu den hauptsächlichen Herkunftsländern einiger von der Schweiz importierter Produkte auf. Die Einfuhren haben sich in den 90er Jahren - insbesondere wegen der starken Schwankungen der Weltpreise für bestimmte Produkte wie Kaffee und Kakao - von Jahr zu Jahr wertmässig erheblich geändert.

Tabelle 7: Wichtigste Bezugsländer für bestimmte von der Schweiz importierte Agrarprodukte, 2004 (in Tausend Franken und Prozent)

\begin{tabular}{lrr}
\hline Frische Schnittrosen für Sträusse & in Tausend Franken & in \% des Gesamtbetrags \\
\hline Industrieländer & $32^{\prime} 142.6$ & 60.5 \\
\hline Ekuador & $8^{\prime} 351.8$ & 15.7 \\
\hline Kenia & $6^{\prime} 782.7$ & 12.8 \\
\hline Simbabwe & $2^{\prime} 697.1$ & 5.1 \\
\hline Tansania & $1^{\prime} 620.7$ & 3.1 \\
\hline Kolumbien & $1^{\prime} 070.5$ & 2.0 \\
\hline Verschiedenes & 440.3 & 0.8 \\
\hline Gesamtbetrag & $\mathbf{5 3} \mathbf{1 0 5 . 7}$ & $\mathbf{1 0 0 . 0}$ \\
\hline Entwicklung der letzten Jahre & Tausend Franken & in Fr./Doppelzentner \\
\hline 1990 & $21^{\prime} 044.2$ & 1748.5 \\
\hline 1995 & $28^{\prime} 067.0$ & 1779.0 \\
\hline 2000 & $58^{\prime} 371.7$ & 1687.5 \\
\hline 2003 & $57^{\prime} 732.3$ & 1515.9 \\
\hline 2004 & $53^{\prime} 105.7$ & 1415.5 \\
\hline
\end{tabular}

\begin{tabular}{lrr}
\hline Bananen & in Tausend Franken & in \% des Gesamtbetrags \\
\hline Costa Rica & $41^{\prime} 617.1$ & 41.3 \\
\hline Kolumbien & $23^{\prime} 534.8$ & 23.3 \\
\hline Ekuador & $16^{\prime} 207.1$ & 16.1 \\
\hline Dominikanische Republik & $6^{\prime} 758.7$ & 6.7 \\
\hline Panama & $5^{\prime} 548.2$ & 5.5 \\
\hline Peru & $2^{\prime} 323.8$ & 2.3 \\
\hline Verschiedene EU-Staaten & $1^{\prime} 792.6$ & 1.8 \\
\hline Verschiedene Entwicklungsländer & $3^{\prime} 057.7$ & 3.0 \\
\hline Gesamtbetrag & $\mathbf{1 0 0} 840.0$ & $\mathbf{1 0 0 . 0}$ \\
\hline Entwicklung der letzten Jahre & in Tausend Franken & in Fr./Doppelzentner \\
\hline 1990 & $95^{\prime} 430.9$ & 125.8 \\
\hline 1995 & $88^{\prime} 961.5$ & 118.6 \\
\hline 2000 & $103^{\prime} 456.2$ & 143.0 \\
\hline 2003 & $105^{\prime} 613.0$ & 146.3 \\
\hline 2004 & $100^{\prime} 840.0$ & 137.1 \\
\hline
\end{tabular}




\begin{tabular}{lrr}
\hline Ungerösteter Kaffee & in Tausend Franken & in \% des Gesamtbetrags \\
\hline Brasilien & $34^{\prime} 943.0$ & 25.5 \\
\hline Kolumbien & $16^{\prime} 736.4$ & 12.2 \\
\hline Mexiko & $13^{\prime} 933.1$ & 10.2 \\
\hline Guatemala & $8^{\prime} 475.9$ & 6.2 \\
\hline Indien & $7^{\prime} 120.8$ & 5.2 \\
\hline Costa Rica & $6^{\prime} 806.1$ & 5.0 \\
\hline Äthiopien & $6^{\prime} 720.3$ & 4.9 \\
\hline Honduras & $5^{\prime} 715.5$ & 4.2 \\
\hline Uganda & $5^{\prime} 331.5$ & 3.9 \\
\hline Kenia & $5^{\prime} 203.8$ & 3.8 \\
\hline Laos & $3^{\prime} 635.6$ & 2.7 \\
\hline Peru & $3^{\prime} 225.3$ & 2.4 \\
\hline Indonesien & $3^{\prime} 155.1$ & 2.3 \\
\hline Andere Länder & $16^{\prime} 001.9$ & 11.7 \\
\hline Gesamtbetrag & $\mathbf{1 3 7} \mathbf{0} 04.3$ & $\mathbf{1 0 0 . 0}$ \\
\hline Entwicklung der letzten Jahre & Tausend Franken & in Fr./Doppelzentner \\
\hline 1990 & $220^{\prime} 881.8$ & 340.0 \\
\hline 1995 & $242^{\prime} 712.7$ & 422.4 \\
\hline 2000 & $232^{\prime} 748.8$ & 349.3 \\
\hline 2003 & $144^{\prime} 036.8$ & 199.4 \\
\hline 2004 & $137^{\prime} 004.3$ & 208.1 \\
\hline
\end{tabular}

\begin{tabular}{lrr}
\hline Kakaobohnen & in Tausend Franken & in \% des Gesamtbetrags \\
\hline Ghana & $22^{\prime} 540.3$ & 32.7 \\
\hline Ekuador & $13^{\prime} 102.9$ & 19.0 \\
\hline Elfenbeinküste & $11^{\prime} 162.7$ & 16.2 \\
\hline Grenada & $1^{\prime} 936.9$ & 2.8 \\
\hline Jamaika & $1^{\prime} 835.1$ & 2.7 \\
\hline Nigeria & $1^{\prime} 743.9$ & 2.5 \\
\hline Venezuela & $1^{\prime} 413.7$ & 2.0 \\
\hline Kuba & $1^{\prime} 203.9$ & 1.7 \\
\hline EU-Staaten & $12^{\prime} 358.6$ & 17.9 \\
\hline Andere Länder & $1^{\prime} 719.9$ & 2.5 \\
\hline Gesamtbetrag & $\mathbf{6 9} \mathbf{9} \mathbf{0 1 7 . 9}$ & $\mathbf{1 0 0 . 0}$ \\
\hline Entwicklung der letzten Jahre & Tausend Franken & in Fr./Doppelzentner \\
\hline 1990 & $53^{\prime} 482.6$ & 250.3 \\
\hline 1995 & $50^{\prime} 757.4$ & 213.5 \\
\hline 2000 & $49^{\prime} 019.1$ & 222.9 \\
\hline 2003 & $92^{\prime} 889.6$ & 351.2 \\
\hline 2004 & $69^{\prime} 017.9$ & 271.7 \\
\hline
\end{tabular}

\begin{tabular}{lrr}
\hline Kakaobutter, Fett und $\mathbf{0} \mathbf{I}$ & in Tausend Franken & in \% des Gesamtbetrags \\
\hline Niederlande & $80^{\prime} 224.2$ & 75.2 \\
\hline Frankreich & $17^{\prime} 539.1$ & 16.4 \\
\hline Andere Industrieländer & $8 ' 267.9$ & 7.8 \\
\hline Entwicklungsländer & 612.9 & 0.6 \\
\hline Gesamtbetrag & $\mathbf{1 0 6} 644.1$ & \\
\hline
\end{tabular}

Quelle: Eidg. Oberzolldirektion, Schweizerische Aussenhande/sstatistik, Statistik nach Waren und Ländern, Bern, Jahrgänge 2004, 2000, 1995 und 1990, für die Tarifposten 0603.1072 (Rosen), 0803.0000 (Bananen), 0901.1100 (Kaffee), 1801.0000 (Kakao). 


\section{C.3.1. Investitionsbestand Ende 2003}

Die Rolle der Direktinvestitionen ist für die Entwicklungsländer wichtig. Die Direktinvestitionen fördern den Technologietransfer und die Schaffung von Arbeitsplätzen. Da sie weniger volatil sind als die Portfolioinvestitionen, stellen sie für die Entwicklungsländer zudem ein relativ stabiles Kapital dar.

2003 beliefen sich die Gesamtbestände der schweizerischen Investitionen im Ausland gemäss den von der Schweizerischen Nationalbank veröffentlichten Zahlen auf 424 Milliarden Franken. Davon waren 43 Prozent in den Ländern der Europäischen Union, 31 Prozent in den übrigen Industriestaaten und 26 Prozent (112,1 Milliarden Franken) in den Entwicklungs- oder Transitionsländern investiert (siehe Grafik 2).

Grafik 2: Aufteilung der schweizerischen Direktinvestitionsbestände im Ausland, Ende 2003 (in Milliarden Franken)
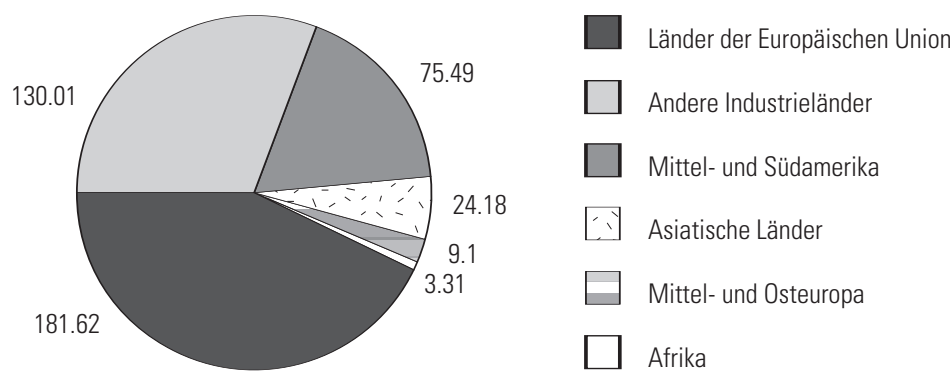

Quelle: Schweizerische Nationalbank, <www.snb.ch>, (unter „Publikationen“, „Zahlungsbilanz und Auslandvermögen der Schweiz", "Direktinvestitionen").

Die Direktinvestitionen im Ausland konzentrieren sich auf eine kleine Anzahl von Entwicklungs- oder Transitionsländern. Andere Länder und Gebiete sind weiterhin von den Investitionen weitgehend ausgeschlossen. Der Investitionsbestand in sämtlichen afrikanischen Ländern machte beispielsweise lediglich 3,31 Milliarden Franken, das heisst 0,8 Prozent der Gesamtinvestitionen (oder nur $3 \%$ der Investitionen in den Entwicklungs- und Transitionsländern) aus. Die Investitionen in Afrika konzentrieren sich vor allem auf Südafrika (1,15 Milliarden Franken), Ägypten (338 Millionen Franken) und Marokko (263 Millionen Franken).

Die Grafik 3 führt die Entwicklungs- und Transitionsländer auf, in denen die Investitionsbestände Ende 2003 am höchsten sind. 50,3 Prozent der Investitionen sind in den Offshore-Finanzzentren ${ }^{1}$ konzentriert, 81 Prozent haben sich auf diese Offshore-Zentren und auf die vierzehn wichtigsten Bestimmungsländer der schweizerischen Investitionen konzentriert.

1 Offshore-Finanzzentren: Anguilla, Bahamas, Barbados, Bermudainseln, Britische Jungferninseln, Jamaika, Kaimaninseln, Montserrat, Niederländische Antillen, Panama, Saint Kitts and Nevis, und seit dem Jahr 2000 Antigua und Barbuda, Belise, Dominica, Grenada, Santa Lucia, Saint Vincent and the Grenadines sowie die Turks- und Caicosinseln. 
Grafik 3: Wichtigste Entwicklungs- und Transitionsländer als Bestimmungsländer der schweizerischen Direktinvestitionen, Bestand Ende 2003 (in Milliarden Franken)

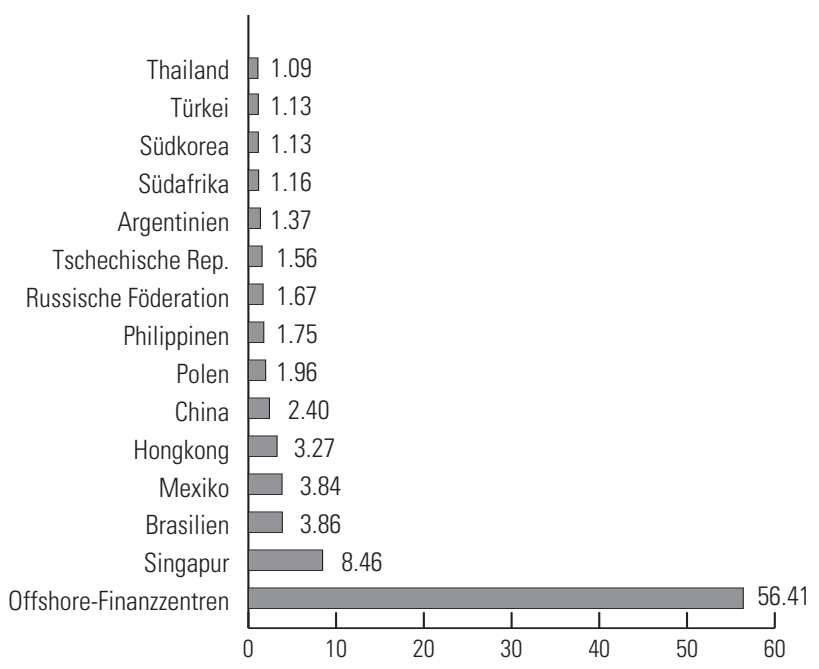

Quelle: Schweizerische Nationalbank, <www.snb.ch>, (unter „Publikationen“, „Zahlungsbilanz und Auslandvermögen der Schweiz", „Direktinvestitionen“).

\section{C.3.2. Flüsse schweizerischer Direktinvestitionen im Ausland im Jahr 2003}

Die Statistiken der Schweizerischen Nationalbank führen die schweizerischen Direktinvestitionsflüsse nach Bestimmmungsländern auf. Die Flüsse sind von einem Jahr zum anderen sehr verschieden. Im Verlauf der letzten Jahre beliefen sie sich 1999 auf 50 Milliarden Franken, im Jahr 2000 auf 75,4 Milliarden und fielen dann 2001 auf 31 Millarden Franken und 2002 auf 12,2 Millarden Franken ab.

2003 erreichten die Flüsse schweizerischer Direktinvestitionen im Ausland 20,4 Milliarden Franken, davon gingen 6 Milliarden in Länder der Europäischen Union und 4 Milliarden in Entwicklungs- und Transitionsländer. Im Vergleich zu den Beträgen der öffentlichen Entwicklungshilfe (2003: 1,7 Milliarden Franken) sind diese Investitionsflüsse somit bedeutend. Jedoch ist hinzuzufügen, dass sie nur in einige Regionen und Länder gehen, wie die Grafik $4^{2}$ zeigt, und dass andere Gebiete von diesen Flüssen vollständig ausgeschlossen bleiben. 2003 waren die Investitionsflüsse nach Afrika weitgehend negativ und wiesen eine Desinvestition von 248 Millionen Franken auf.

2 Der Gesamtbetrag der Investitionsflüsse für die in Grafik 4 aufgeführten Länder ist höher als die Summe der Gesamtinvestitionen in den Entwicklungs- und Ttransitionsländern. Dies erklärt sich aus der Tatsache, dass die Investitionsflüsse im Jahr 2003 für viele Entwicklungsländer negativ sind (Desinvestitionen). 
Grafik 4: Wichtigste Entwicklungs- und Transitionsländer als Bestimmungsländer der schweizerischen Direktinvestitionsflüsse, 2003 (in Millionen Franken)

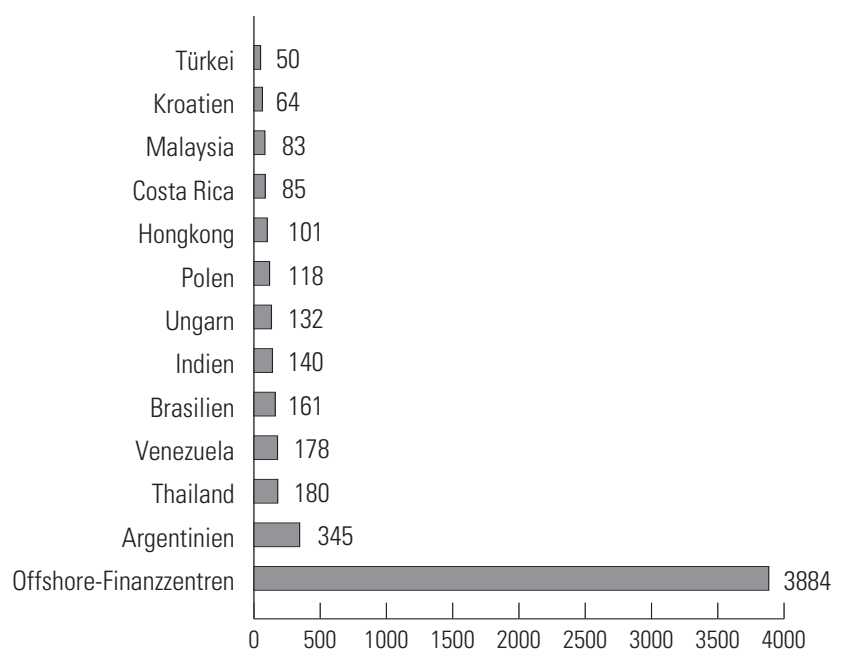

Quelle: Schweizerische Nationalbank, <www.snb.ch>, (unter „Publikationen“, "Zahlungsbilanz und Auslandvermögen der Schweiz", "Direktinvestitionen“).

\section{$\square$ Anmerkungen}

Die Schweizerische Nationalbank trägt die Informationen über die schweizerischen Direktinvestitionen im Ausland zusammen, wenn ein Anleger sich mit mindestens 10 Prozent am Kapital eines Unternehmens im Ausland beteiligt oder eine Tochtergesellschaft bzw. eine Filiale im Ausland gründet. Die in einem Jahr registrierten Investitionsflüsse erfassen Beteiligungen an Eigenmitteln (Gründungen, Erwerbungen, Verkäufe, Kapitalerhöhungen), Konzernkredite sowie reinvestierte Erträge. Es handelt sich um Nettoflüsse, wobei eine negative Zahl einen Kapitalrückfluss anzeigt. Der Bestand am Ende eines Jahres plus dem Fluss des folgenden Jahres entspricht nicht unbedingt dem Bestand am Ende des folgenden Jahres, da sich die Bestandsänderungen auch aus Faktoren ergeben, die nicht in den Kapitalflüssen erscheinen (Entwicklung des Wechselkurses, Änderung der Buchhaltungsmethoden); im Ausland finanzierte Erwerbungen geben ebenfalls nicht zu Kapitalflüssen Anlass. Die Statistiken versuchen, soweit wie möglich das Land des zuletzt Begünstigten zu berücksichtigen, was aber nicht immer möglich ist, wie zum Beispiel im Fall der OffshoreFinanzzentren. 


\section{C.4. Öffentliche Entwicklungshilfe der DAC-Länder und der Schweiz}

\section{$\square$ Langfristige Entwicklung der öffentlichen Entwicklungshilfe der DAC-Länder und der Schweiz ${ }^{3}$}

Die öffentliche Entwicklungshilfe der Mitgliedsländer des DAC erreichte 2003 den Rekordstand von 69 Millionen Dollar. Der vorherige Rekordwert war 1992 volumenmässig mit einem Gesamtbetrag der Hilfe von 60,9 Milliarden Dollar erreicht worden (siehe Grafik 5).

In Prozent des Bruttonationaleinkommens (BNE) ausgedrückt, fällt diese Erhöhung jedoch bescheidener aus, da die öffentliche Entwicklungshilfe der DACLänder von 0,22 Prozent des BNE 2001 auf 0,25 Prozent 2003 anstieg, was erheblich unter dem von den Vereinten Nationen festgesetzten Ziel von 0,7 Prozent der Hilfe und sogar unter dem im Zeitraum 1980-1992 durchschnittlich verzeichneten Satz von 0,33 Prozent liegt (siehe Grafik 7).

Der Anstieg der öffentlichen Entwicklungshilfe in den letzten Jahren lässt sich zum grossen Teil auf folgende Faktoren zurückführen:

๖ 74 Prozent der Erhöhung der öffentlichen Entwicklungshilfe von 2002 auf 2003 sind durch die Auswirkungen der Inflation und vor allem durch den Rückgang des Aussenwerts des Dollars bedingt.

๖ Die bilateralen Spenden haben für einige Länder stark zugenommen, darunter zusätzliche 1,9 Milliarden Dollar an bilateraler öffentlicher Entwicklungshilfe allein für den Irak.

๖ Die Nettospenden in Form von Schuldenerlassen stiegen ihrerseits um 2,1 Milliarden Dollar an. Die Schuldenerleichterungsmassnahmen beliefen sich 2003 auf 9,4 Milliarden Dollar, das heisst rund 14 Prozent der gesamten öffentlichen Entwicklungshilfe der DAC-Länder. Die Schuldenerleichterungsmassnahmen Frankreichs überstiegen 2,9 Milliarden Dollar, was über 40 Prozent der öffentlichen Entwicklungshilfe dieses Landes entspricht. Für die Schweiz betrugen diese Massnahmen 75 Millionen Dollar, das heisst 5,8 Prozent der gesamten öffentlichen Entwicklungshilfe.

Die öffentliche Entwicklungshilfe der Schweiz erreichte 2003 ebenfalls ein Rekordniveau, wobei sie den 1992 von der Schweiz anlässlich ihres Beitritt zu den Bretton-Woods-Institutionen gezahlten Betrag öffentlicher Entwicklungshilfe überstieg (siehe Grafik 6). Dies erklärt sich zum Teil durch eine von 2002 auf 2003 verschobene Zahlung an die Internationale Entwicklungsorganisation (IDA).

3 Quellen: OECD, DAC-Statistiken und Kommentar der OECD zu den endgültigen Daten der öffentlichen Entwicklungshilfe für 2003, <www.oecd.org/cad>. 
Grafik 5: Entwicklung der Hilfe der DAC-Länder, 1987-2003 (in Millionen Dollar)

$\square$ ÖEH = öffentliche Entwicklungshilfe

$\square$ ÖH = öffentliche Hilfe

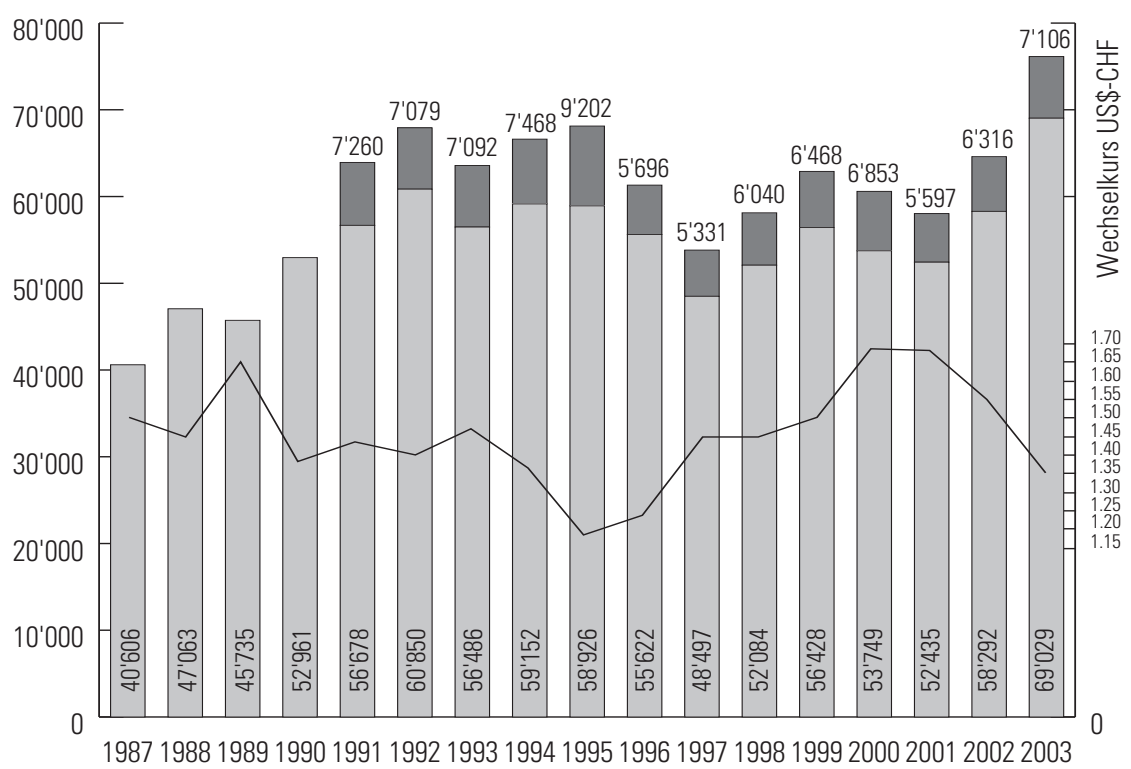

Quelle: OECD, DAC-Statistiken, <www.oecd.org.cad>.

\section{Grafik 6: Entwicklung der Hilfe der Schweiz, 1987-2003 (in Millionen Dollar)}

$\square$ ÖEH = öffentliche Entwicklungshilfe

$\square$ ÖH = öffentliche Hilfe

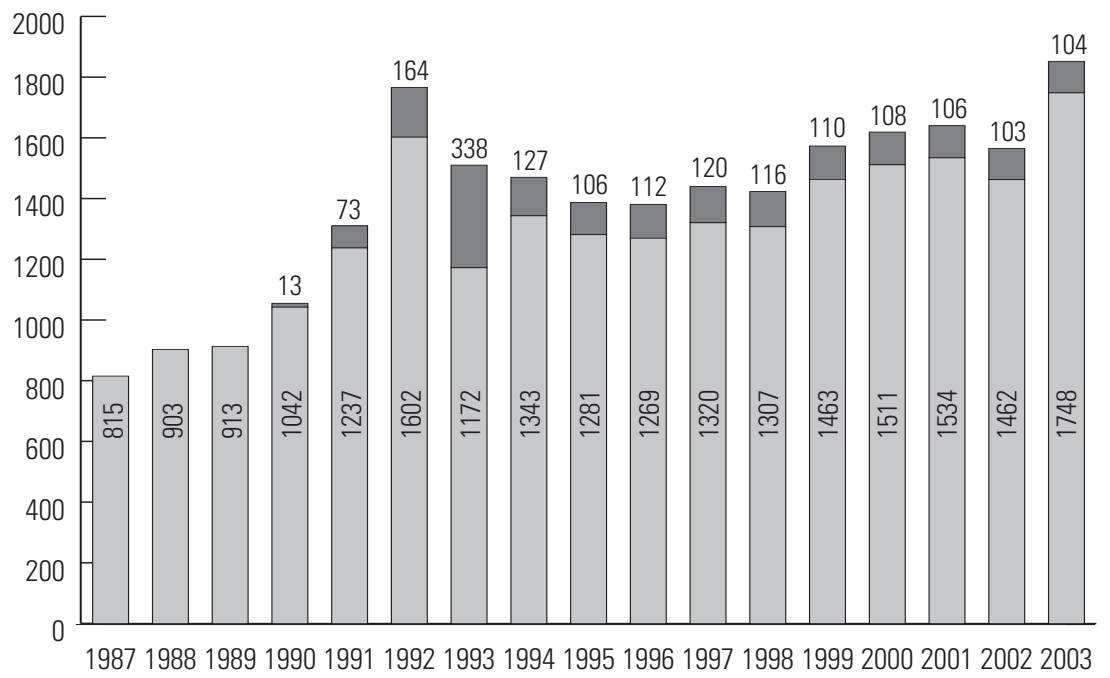

Quelle: OECD, DAC-Statistiken, <www.oecd.org.cad>. 
Grafik 7: Entwicklung der öffentlichen Entwicklungshilfe der DAC-Länder und der Schweiz, 1986-2003 (in Prozent des BNE)

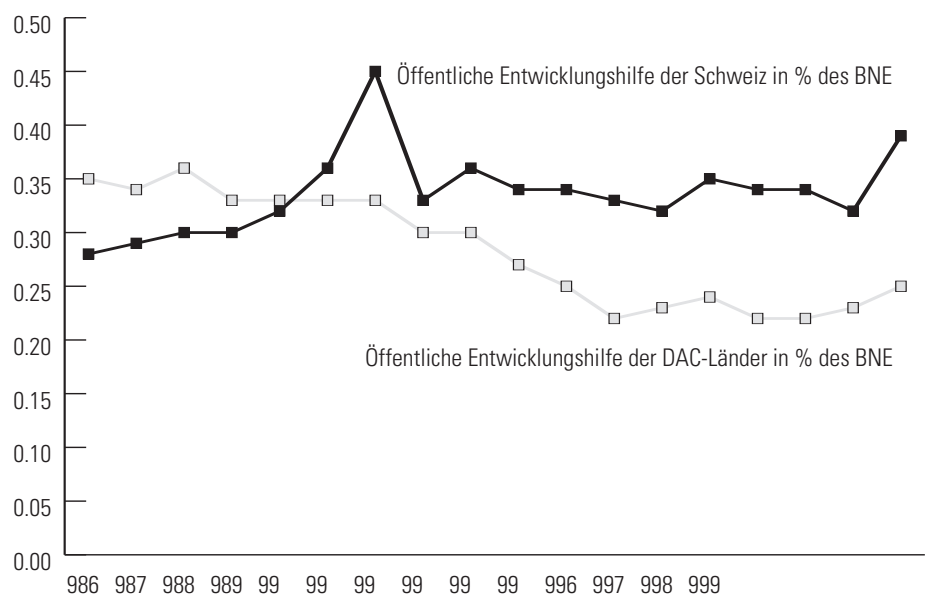

Quelle: OECD, DAC-Statistiken, <www.oecd.org.cad>.

Die Hilfe der Schweizer Kantone und Gemeinden wird im Aggregat „öffentliche Hilfe“ oder „öffentliche Entwicklungshilfe“ berücksichtigt, jedoch wird sie hier getrennt aufgeführt, um die Entwicklung im zeitlichen Verlauf feststellen zu können.

Die den Kurven der Kantone, der Gemeinden und der NRO zugrunde liegenden Zahlen werden anhand von Jahresumfragen erhalten, bei denen der Prozentsatz der Rückantworten ein für das Ergebnis signifikanter Faktor ist. Sie müssen somit als Minimalwerte angesehen werden.

\section{Grafik 8: Entwicklung der öffentlichen Entwicklungshilfe/Hilfe und der privaten Hilfe der NRO für die Schweiz, 1989-2003 (Index 1989 : 100)}

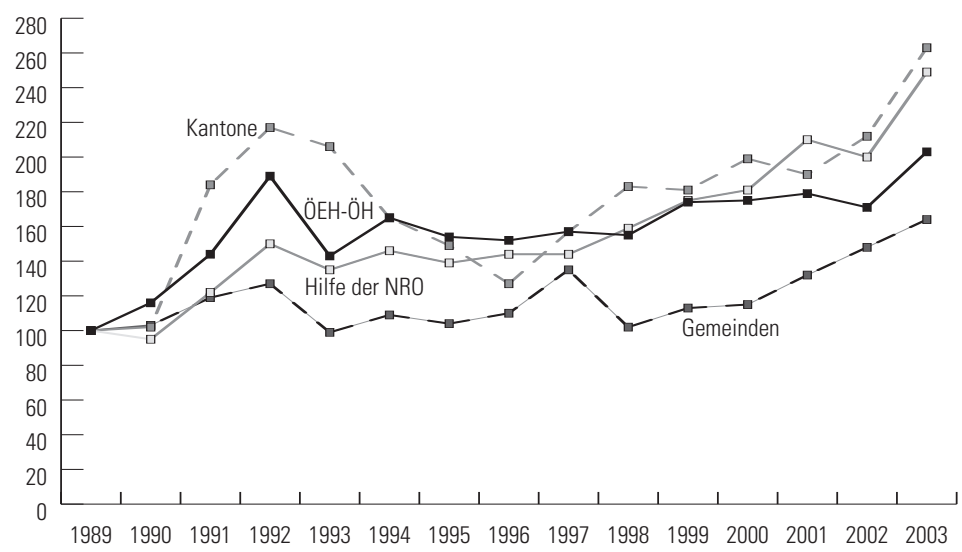

Quellen: Swissaid, Aide suisse aux pays en développement, 1988-1990, Bern; iuéd/DEZA, Schweizerische Hilfe für Entwicklungsländer und Oststaaten, Publikationen von 1991 bis 1997, Genf; Aide suisse aux pays en développement et aux pays en transition, 1998-2000, Genf; DEZA, Statistischer Dienst, Aide de la Suisse aux pays en développement et aux pays en transition, Statistiques 2001, 2002, 2003, Bern. 
Die Nichtregierungsorganisationen finanzieren Entwicklungshilfeprojekte gänzlich oder teilweise mit ihren Eigenmitteln. Die Beträge dieser privaten Hilfe beliefen sich 2003 auf 377 Millionen Franken für die Entwicklungsländer (Teil I der DAC-Liste) und auf 15 Millionen Franken für die Transitionsländer (Teil II der DAC-Liste) (siehe Liste am Schluss des Jahrbuchs).

\section{Grafik 9: Entwicklung der privaten Hilfe der NRO in der Schweiz, 1986-2003 (in Millionen Franken)}

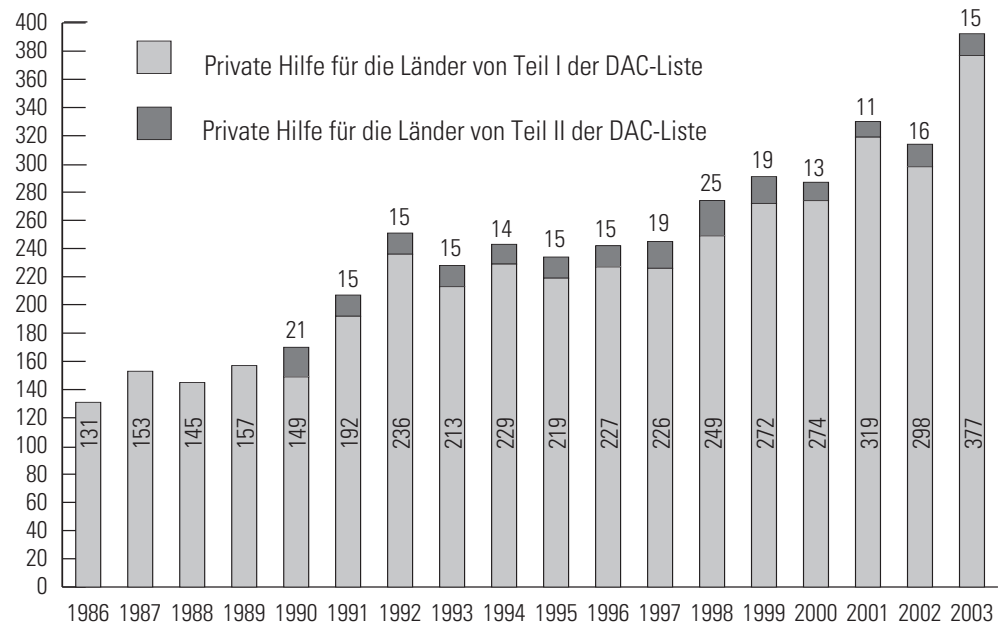

Quellen: Swissaid, Aide suisse aux pays en développement, 1988-1990, Bern; iuéd/DEZA, Schweizerische Hilfe für Entwicklungsländer und Oststaaten, Publikationen von 1991 bis 1997, Genf; Aide suisse aux pays en développement et aux pays en transition, 1998-2000, Genf; DEZA, Statistischer Dienst, Aide de la Suisse aux pays en développement et aux pays en transition, Statistiques 2001, 2002, 2003, Bern. 